Revue d'histoire de l'Amérique française

FBS REVUE D.HISTOIRE DE L'AMÉRIQUE FRANÇAISE

\title{
Les études historiques sur Saint-Domingue depuis 1938
}

\section{Gabriel Debien}

Volume 4, numéro 1, juin 1950

URI : https://id.erudit.org/iderudit/801629ar

DOI : https://doi.org/10.7202/801629ar

Aller au sommaire du numéro

Éditeur(s)

Institut d'histoire de l'Amérique française

ISSN

0035-2357 (imprimé)

1492-1383 (numérique)

Découvrir la revue

Citer ce document

Debien, G. (1950). Les études historiques sur Saint-Domingue depuis 1938.

Revue d'histoire de l'Amérique française, 4(1), 135-142.

https://doi.org/10.7202/801629ar d'utilisation que vous pouvez consulter en ligne.

https://apropos.erudit.org/fr/usagers/politique-dutilisation/ 


\section{BIBLIOGRAPHIE}

\section{LES ÉTUDES HISTORIQUES SUR SAINT-DOMINGUE DEPUIS 1938}

\section{I - Biblographies}

$\mathrm{C}_{\mathrm{ABON}}, \mathbf{R}^{\mathrm{d}} \mathrm{P}$. "History of Haiti journalism", Proceadings of the Amer. Antiq. Society (April 1939), Worcester, Mass. 87 p.

Bibliographie des journaux de Saint-Domingue conservés dans la Bibliothèque du Séminaire - Collège Saint-Martial à Port-auPrince. Traduction d'articles parus, d'avril à novembre 1919, dans la Petite Revue Hebdomadaire que publiait à Paris, M. Doret, Haïtien. Debien, G. "Notes sur les journaux de Saint-Domingue", Rev. d'Hist. et Géog. d'Haiti, 14, no 49 (avril 1943): 6-16.

Debien, G. "Notes bibliographiques sur l'histoire de Saint-Domingue: I, Histoire médicale", Rev. d'Hist. et Géog. d'Haïti, 14, no 48 (janvier 1943): $25-32$.

Idem. "Notes bibliographiques sur l'histoire de Saint-Domingue: II, l'Expédition de 1802." Id.: 32-42.

Idem. "Notes bibliographiques sur l'histoire de Saint-Domingue: III, Le Soulèvement des esclaves (août-septembre 1791)." Id., 17, no 62 (juillet 1946): $41-57$.

Debien, G. "Pour connaître un type de fortune: les archives de quelques familles de planteurs antillais", Ann. d'hist. écon. et soc. (septembre 1938): $424-429$.

Debien, G. "Archives de planteurs: I, Papiers Galbaud du Fort; II, Papiers Croisœuil; III, Papiers Bréda, Noé, Butler, d'Héricourt et Polastron; IV, Papiers la Merveillère", Rev. d'Hist. et Géog. d'Haĩti, 12, no 40 (janvier 1941): $39-51 ; 14$, no 49 (avril 1943): $17-23 ; 17$, no 61 (avril 1946): $31-45$. Id.: $21-28$.

Debien, G. "Documents dispersés. Chronique des ventes", $i d$., 12, no 42 (juillet 1941): 21-50.

Debien, G. "Les travaux d'histoire sur Saint-Domingue, 1938-1946. Essai de mise au point", Revue d'Histoire des Colonies (1947): 31-86.

Debien, G. "Archives de plantations des Antilles", $B^{\text {in }}$ Soc. hist. de Nantes (1947), $29 \mathrm{p}$. 
Duvivier, Ulrick. Bibliographie générale et méthodique d'Haiti. 2 vol., Port-au-Prince, 1941. 318 et 411 p.

[ Assez peu ordonnée, non critique, très utile ]

MaUrel, MLle B. "Revue des livres, manuscrits et autres documents rares relatifs à Saint-Domingue et dont le dépôt se trouve à la Bibliothèque Nationale à Paris." Rev. d'Hist. e Géog. d'Haïti,12, no 38 (juillet 1940): $39-43$.

Ragatz, Lowel Joseph. "Early French West Indians Records in the Archives Nationales", Inter American Bibliog. Review, I, no 3 (1942).

\section{II - HistoIRE GÉNÉRALE}

Beaudza, L. La formation de l'armée coloniale. Paris, Fournier, 1939. $617 \mathrm{p}$.

Cook, M. "Literary Contribution of the French West Indian", Journal Negro Hist. XXV (octobre 1940): $520-530$.

Crouse, N. M. French Pionners in the West Indies (1624-1664). Now York, 1940. 294 p.

Idem. French Struggle for the West Indies (1665-1713). New York, 1943. $324 \mathrm{p}$.

David, P. Sur les rives du passé. Choses de Saint-Domingue. Paris, s.d. [ 1947 ]. 230 p.

Debien, G. "L'esprit d'indépendance chez les colons de Saint-Domingue au XVIII' siècle et l'appel aux Anglais on 1793", Rev. d'Hist. et Géog. d'Haïti, 17, no 63 (octobre 1946): $1-46$.

EuIE, L.-E. Histoire d'Haïti. 2 vol., Port-au-Prince, 1944-1945. 258 et $305 \mathrm{p}$.

Gipson, L.H. Zones of International Friction: Canada, the West Indies, Indies, 1743-1754. New York, 1942. XLVIII, $352 \mathrm{p}$.

JANIN, $\mathbf{R}^{\mathrm{d}} \mathrm{P}$. La religion aux colonies françaises sous l'ancien régime (de 1626 à la Révolution), Paris, s.d. [1947], 234 p.

Leyburn, James G. The Haytian People. New Haven, 1941. 342 p.

Nicaise, P. Les Assemblées délibérantes dans les anciennes colonies françaises. Paris, 1940. $145 \mathrm{p}$.

Price-Mars, Dr. "L'Afrique noire et ses peuples." Introduction au cours d'Africologie à l'Institut d'ethnologie. Ré̀. d'Hist. et Géog. d'Haiti, 13, no 44 (janvier 1942): $30-44$.

RoBerts, A.W. Les Français aux Indes Occidentales. Traduction par Antoine Coffard. Montreal, 1946.

Roberts, A.W. The Caribbean. The Story of Our Sea Destiny. Indianapolis, 1940. $361 \mathrm{p}$.

SAINT-VICtoR, J.-B. Deux concepts d'indépendance à Saint-Domingue. Port-au-Prince, 1944. $306 \mathrm{p}$.

Williams, E. The Negro in the Caribbean Washington, the Associates in Negro Folk Education. 1942. 119 p. 


\section{III - Traite et esclavage}

Berbain, Mlle S. Etudes sur la traite des noirs au golfe de Guinée. Le comptoir français de Juda (Ouidah) au XVIII $I^{e}$ siècle. Mém. Inst. Franc. Afrique Noire. Paris, 1942. 126 p. 6 pl.

Debien, G. "Les papiers d'un capitaine négrier", Rev. d'Hist. et Géog. d'Haiti, 12, no 43 (octobre 1941): 30-50.

Ducasse, A. Les négriers ou le trafic des esclaves. Paris, [ 1948 ]. [ Bibliographie sommaire ].

Lokke, C.L. "Le plaidoyer de Malouet en faveur de l'esclavage en 1789", An. Hist. Rév. Fr., mai 1938.

Martin, Gaston. Histoire de l'Esclavage dans les colonies françaises. Paris, 1948. Collection "Colonies et empires".

Pfister, A. Essai sur le code noir et la condition juridique des esclaves dans l'ancien droit franşais. Bordeaux, 1946. 70 pages dactylographiées. Thèse de droit.

Mc Cloy, S.T. "Negroes and mulattoes in eighteenth century France", Journ. Negro Hist., XXX (july 1945): 276-292.

Rinchon, $\mathbf{R}^{\mathbf{d}}$ P.D. Le trafic négrier d'après les livres de commerce du capitaine gantois P.I. Liévin van Alstein. Bruxelles, Paris, 1938.

\section{iV - Peuplement et colonisation Plantation et colons} (XVIIe ET XVIIIO SIÈCLES)

Begougn-Demeaux, M.Mémorial d'une famille du Havre, I: les fondateurs, choses et gens du XVIII e siècle en France et à Saint-Domingue. Lo Havre, 1948. 137 p.

Chevalier, G.A. "Etude sur la colonisation française en Haiti. Origines et développement des propriétés Collette." Rev. d'Hist. et Géog. d'Haïti, 9, no 31 (octobre 1938): $11-46 ; 10$, no 33 (avril 1939): 17-41. [ Caféières au Gros-Morne et à Jean-Rabel].

Debign, G. "Etablissement d'une caféière à Saint-Domingue", B in . Soc. Arch. Nantes, 80 (1940): 141-152.

Debien, G. "Une indigoterie à Saint-Domingue à la fin du XVIIIe siècle", Rev. d'hist. des Colonies (1940-1946): 1-49.

Debien, G. "Le plan et les débuts d'une caféière à Saint-Domingue. La plantation la Merveillère aux Anses-à-Pitre (1789-1792)", Rev. d'Hist. et Géog. d'Haïti, 14, no 51 (octobre 1943): 12-32.

Debien, G. Une plantation de Saint-Domingue. La sucrerie Galbaud du Fort (1690-1802). Le Caire, 1941. 136 p.

Idem. "Comptes, profits, esclaves et travaux de deux sucreries de SaintDomingue (1774-1798), Rev. d'Hist. et Géog. d'Haïti, 15, no 55 (octobre 1944): $1-60 ; 16$, no 56 (janvier 1945): $1-60$. 
Idem. "A Saint-Domingue avec deux jeunes économes de plantation (1774-1788)", Id., 16, no 58 (juillet 1945): $1-80$.

Idem. 'Gouverneurs, magistrats et colons. L'opposition parlementaire et coloniale à Saint-Domingue (1763-1769)", Id., 16, no 59 (octobre 1945); 17, no 60 (janvier 1946).

Debien, G. Le peuplement deis Antilles françaises au XVII siècle. Les engagés partis de la Rochelle (1683-1715). Le Caire, 1942. 223 p.

Idem. "Contrats d'engagements de Marchois pour les îles (XVII॰ ot XVIII" siècles)", Mém. Soc. Sc. Creuse, XXVII (1939-1940): 49-50.

Idem. "Aux origines de quelques plantations des quartiers de Léogane et du Cul de Sac (1680-1715)", Rev. d'Hist. et Géog. d'Haiti, 18, no 64 (janvier 1947): 10-78.

Dumon. Une carrière de commissaire de la Marine au XVIII siècle, François de Magny. Contribution à l'étude du corps des officiers de plume. Lyon, 1940. $296 \mathrm{p}$.

[ sur un épisode de l'intendance de Clugny à Saint-Domingue ]

Hesse, Mme G. "Un établissement français à Saint-Domingue au XVIII" siècle", de France, (1er sept. 1938): 278-301. [indigoterie Belin, à Léogane]

Jounaud, L. Mcurs limousines au XVIII $I^{e}$ siècle. Les Pas Perdus. Limoges, 1938.

Cf. le chapitre: Fortune d'Amérique: 151-207.

[ Histoire du colon Antoine de Bessas, à Saint-Domingue, de 1784 à 1799].

Laurent, M. "Port-au-Prince. Ses origines, ses édifices, ses places publiques, ses rues...", Le Document, organe de la librairie d'histoire d'Haiti, no 1 (janvier 1940) : 37-65; no 2 (février 1940) : 225-232; no 4 (avril 1940): $318-326$.

Laurent, G.M. “Coup d'œil sur le système des engagés”, Rev. d'Hist. et Géog. d'Haïti, 19, no 69: 22-30.

LugGo, C. "Jean-Baptiste Lepers, Histoire civile, morale et naturelle de l'isle de Saint-Domingue", Bol. Arch. Gen. Nac. Ciudad Trujillo, République Dominicaine, sept. 1946.

Marion, Jean. "Un Nantais à Saint-Domingue (1756-1792)", $B^{\text {in }}$ Soc. Arch. Nantes, t. 78 (1938): 114-133.

[Pierre Guiau, tonnelier devenu planteur caféier au-dessus de Portau-Prince; d'après sa correspondance ]

MontFont, P. "En marge de la traite des noirs: 1785. Joseph Fouche, propriêtaire à Saint-Domingue", $B^{\text {in }}$ Soc. Arch. Nantes (1941), t. 81.

Moreau de SaINT-MÉry. "Les impôts et les mesures agraires en 1783", p. p.Em. Rodriguez Demorizi. Bol. Camera oficial Espan. de Comercio $e$ Ind. Ciudad Trujillo, avril 1947.

Neveu, Jacques, "Une plantation d'indigo à Saint-Domingue en 17301740”, Mém. Soc. Agric. d'Angers, 7ème série, XIX (1945): 17-39. 
Pressorr, Ch. F. "La gourde, monnaie des colonies françaises d'Amérique: Guadeloupe, Louisiane, Saint-Domingue", Rev. d'Hist. et Géog. d'Haïti, 19 , no 67 (octobre 1947): 70-77, 88-90.

Pressoir, D $\mathrm{D}^{\mathrm{r}}$ C. "Quelle est l'origine du mot gourde?", Id., 19, no 66 (juillet 1947): 67-77.

Roвillot, J. "Monbars l'imaginaire", $B^{\text {in }} C^{t \varepsilon}$ Trav. hist. Sect. géog. (1942): $71-78$.

\section{V - Les ports de France et Saint-Domingue}

Debien, G. "Aperçu sur la viè à Nantes (1791-1796) d'après une correspondance de Saint-Domingue", $B^{i n}$ Soc. Arch. Nantes (1940), t. 80.

Idem, Le commerce nantais et la perte de Saint-Domingue d'après une correspondance de la maison Lebourg (1784-1800). Port-au-Prince, 1944. $69 \mathrm{p}$.

HalgouËt, Vte H. DU. "Nantes. Ses relations commerciales avec les îles d'Amérique au XVIIIe siècle. Ses armateurs", Mém. Soc. hist. et arch. de Bretagne, XX (1939), IV, 294 p. ill.

Idem. Au temps de Saint-Domingue et de la Martinique d'après la correspondance des trafiquants maritimes. Rennes, 1941. 130 p. tiré à part des Mém. Soc. hist. et arch. de Bretagne, 1941.

\section{VI - La Révolution ì Saint-Domingue}

Cook, M. "Julien Raimond", Journ. Negro Hist. XXVI (1941): 139-170. Chevalier, G.A. "Un colon de Saint-Domingue pendant la Révolution: Pierre Collette, planteur de Jean-Rabel", Rev. d'Hist. et Géog. d'Haïti, janvier-avril-juillet-octobre 1940, janvier 1941.

David, P. édit. "Journal inédit tenu par le colon Gamot des événements accomplis à Saint-Domingue à partir de 1790", le Document, no 3: $172-208$.

Laurent, M. "La femme dans la guerre de l'Indépendance", le Document, no 2: 77-129.

[ Laurent ], M. "Bases constitutionnelles publiées le 28 mai 1790 par la grande assemblée coloniale de Saint-Domingue", le Document, no 3: $261-265$.

[ Laurent, M. éd.] "Blanchelaude ex-gouverneur de Saint-Domingue, convaincu de trahison par ses propres moyens justificatifs", le Document, no 3: $208-224$.

LaURent, M. "Une des premières réclamations des affranchis à SaintDomingue (1789)", le Documant, no 3 (1946): 161-171.

Laurent, M. "La légion franche de cavalerie des Américains et du Midy, communément appelée la Légion noire", Rev. d'Hist. et Géog. d'Haîti, 11, no 34 (juillet 1939): $1-9$. 
LoKke, C.L. "Malouet and the Saint-Domingue mulatto question in 1793", Journal Negro Hist. XXIV (octobre 1939): 381-389.

Idem. "London Merchant Interest in Saint-Domingue Plantations of the Emigrés", $A m . H^{\text {al }}$ Rev. (july 1939): 795-802.

Idem. "New Light on London Merchant investment in Saint-Domingue", Hisp. Am. Hal Rev. (november 1942): 670-676.

Mangonìs, E. "Le Colon Mallet, officier de l'armée révolutionnaire, signataire de l'acte d'indépendance. Sa descendance", Rev. d'Hist. et Géog. d'Haïti, 91, no 30 (juillet 1938): 19-45.

MaUrel, $M^{\text {lle }}$ B. Saint-Domingue et la Révolution française. Les représentants des colons en France de 1789 à 1795. Paris, 1944. 44 p. Extr. Rerue historique, 1944.

Navarre, $R^{d}$ P., Un essai de réorganisation du service de santé à SaintDomingue en 1792-1793. Clermont-Fenaud, 1943. Thèse dactylographiéé.

Idem. La révolte de l'escadre française de Saint-Domingue, le 20 juin 1793, ses causes et ses conséquences. Id., thèse principale dactylographióe.

Nemours, Gal. "Le projet de Constitution de 1790 et la Constitution de 1801", le Document, no 4: 326-331.

\section{VII - TOUSSAINT LOUVERTURE}

Bissainthe, Max. "Une lettre de Toussaint Louverture", [ Port Républicain, 16 avril 1800, au commodore Silas Talbot, à bord de la Constitution ] Rev. d'Hist. et Géog. d'Haïti (juillet 1947): 63-66.

Brutus, T. Rançon du génie ou la leçon de Toussaint Louverture, 2 vol., Port-au-Prince, 1945.

Daroudel, L. "La fortune de Toussaint Louverture et Stephen Girard". Rev. d'Hist. et Géog. d'Haïti, 14, no 50 (juillet 1943): 1-67.

Debien, G. "A propos du trésor de Toussaint Louverture", Id., 17, no 62 (juillet 1946): $30-40$.

Korngold, R. Citizen Toussaint. Boston, 1944. 368 p.

Laurent, M. Erreurs et vérités dans l'histoire d'Haïti. Port-au-Prince, 1945. $383 \mathrm{p}$.

[ Laurent, M. éd.] "De l'organisation de la révolte des esclaves du nord (1791)", le Document, no 3: 245-261.

[ Au texte de C. Ardouin, réimprimé, sont jointes trois lettres de Toussaint Louverture, des 4 et 15 octobre 1791, du 24 germinal au VII. ]

[ Laurent, M. éd. ] "Mémoire de Toussaint Louverture écrit par lui-même", le Documont, no 4: 267-317.

LAURENT', M. "Toussaint Louverture et l'indépendance de Saint-Domingue. Explication par les documents des traités secrets entre Toussaint Louverture, les États-Unis d'Amérique et l'Angleterre", le Documerit, no 5 (mai 1946). 147 p. 
Nemours, $\mathrm{G}^{\mathrm{al}}$. Histoire des relations internationales de Toussaint Louverture. Port-au-Prince.

Nemours, Gal. Histoire de la famille et de la descendance de Toussaint Louverture. Port-au-Prince, 1941.

Nemours, Gal. "La tentation et la mort du Christ noir: Toussaint Louverture", Rev. d'Hist. et Géog. d'Haïti, 15, no 54 (juillet 1944): 20-35.

Nemours, Luc. "Pour quelles raisons Toussaint Louverture est-il passé des Espagnols aux Français?", An. hist. Rév. fran. (av.-juin 1948): $166-171$.

[ Rosset, commissaire principal de la marine ], "Notice historique sur les événements... de floréal an IV au ler brumaire an VII", Rev. d'Hist. et Géog. d'Haïti, 13, no 45 (avril 1942): 37-50.

\section{VIII - L'EXPÉDITION DE 1802}

Bonnette, P. "Le Calvaire médical de Saint-Domingue et de la Guadeloupe", Hippocrate, (octobre 1938): 470-490.

[ d'après les Archives du Val de Grâce ]

Brutus, T.C. L'homme d'airain. Etude monographique sur Jean-Jacques Dessalines, fondateur de la nation haïtienne. v I. Port-au-Prince, 1946.

Debien, G. "Autour de l'expédition de Saint-Domingue. Les espoirs d'une famille d'anciens planteurs", Revue d'Hist. et Géog. d'Haïti, 13, no 47 (octobre 1942): $1-95$.

Idem. "Une lettre sur l'expédition de 1802", Id., 14, no 49 (avril 1943): $24-27$.

James, C.L.R. "La guerre d'indépendance à Haïti", Revze internationale (juin 1947).

Laurent, M. [ Leclere, Toussaint Louverture et Christophe ], le Document, no 4: $344-362$.

Laurent, M. "Lettres révélant le traitement infligé aux officiers de l'étatmajor de Toussaint après sa déportation", le Document, no 4: 331-344.

Navarranne, P. Une expédition coloniale ayant tourné à la catastrophe épidémiologique: Saint-Domingue. Marseille, 1943. [thèse médecine Montpellier ]

Nemours, Gal . "Histoire militaire d'Haïti. Saint-Domingue place d'armes française dans les Antilles (fin du XVII', début du XVIII ${ }^{\bullet}$ siècle)", Revue d'Histoire de l'Amérique française, no 1 (juin 1947): 94-101.

\section{IX - Dispersion des colons}

Blanco, E.T. Los tres ataques britanicos a la Ciudad de Sin Juan Bautista de Puerto Rico: Drake, 1595, Clifford, 1597; Abercrombie, 1797, San Juan Bautista, 1947.

[ rôle des réfugiés français de Saint-Domingue, lors du siège de 1797 ] 
Chateaubriand, G. de. "Les réfugiés de Saint-Domingue. Quelques notes et documents, 1793-1914", Mém. Acad. Angers (1942): 78-103.

ChILd, Miss F.S. Freinch Refugee Life in the United States, 1790-1800. An American Chapter of the French Revolution. Washington, Baltimore, 1940.

Debien, G. "Joseph-Desiré de Messemé (1751-1821), son émigration, la vente et le rachat de ses biens", $B^{\text {in }}$ Soc. Antiq. Ouest, $3^{\mathbf{e}}$ série, T. XIII: $82-105$.

Hardridge, W.C. "The Saint-Domingan Refugees in New Jersey", Proc. New Jersey Hist. Soc. 1944.

Lokke, C.L. et Debien, J. "L'expédition d'Egypte et les projets de cultures coloniales", $B^{\text {in }} S^{t \ell}$ roy. de géog. d'Egypte, XX (1940): 337-356.

Murray, E. "French Refugees of 1793 in Pennsylvania", Proc. Am. Philos. Soc. 87 , no 5 (1944); $387-393$.

Moreau de SaInT-MÉry, American Journey (1793-1798), trad. K. Roberts. New York, 1947.

Pèrez de la Riva, Fr. El Café: Historia de su cultivo y explotacion en Cuba. Biblioteca de Hist. Filosofia y Sociol. 16, La Havane, 1944.

Ruskowski, R ${ }^{\mathrm{d}}$ P.F. French Emigré Priesto in United States (1791-1815) Washington, 1940. $140 \mathrm{p}$.

\section{$\mathrm{X}$ - L'INDÉPENDANCE}

Hughes, H.B.L. "British Policy towards Haiti (1801-1805) as shown in the Nugent Papers", Canada $H^{a l}$ Rev., 25 (1944): 397-408.

Lee Montague, L. Haiti and United States. Durham, N. C. 1940.

Logan, R.W. The Diplomatic Relations of the United States with Haiti, 1776-1891. Chapel Hill, Univ. of N. C. Press, 1941.

PadgetT, J.A. "Diplomats to Haiti and their Diplomacy", Journal Negra Hist. (july 1940).

Perkins, D. The Monroe Doctrine (1826-1862). Baltimore, 1940.

Pressoir, Dr C. "Genèse d'une Nation", Rev. d'Hist. et Géog. d'Haïti, 19, no 69 (avril 1948): 31-55.

Price-Mars, $D^{r}$ "Les relations diplomatiques entre les États-Unis et Haiti" (1717-1804), Rev. d'Hist. ot Géog. d'Hä̈ti, 15, no 53 (avril 1941): $1-14$.

Robertson, W.S. France and Latin American Independance. Baltimore, 1939.

Gabriel Debien, Le Caire, Egypte. 